\title{
Análise espectral usando a transformada contínua de wavelet para pulsações magnéticas Pc5 em áreas magneticamente conjugadas
}

Camacho E., Benyosef L. e Mendes O.

Copyright 2019, SBGf - Sociedade Brasileira de Geofísica

This paper was prepared for presentation during the $16^{\text {th }}$ International Congress of the Brazilian Geophysical Society held in Rio de Janeiro, Brazil, 19-22 August 2019.

Contents of this paper were reviewed by the Technical Committee of the $16^{\text {th }}$ International Congress of the Brazilian Geophysical Society and do not necessarily represent any position of the SBGf, its officers or members. Electronic reproduction or storage of any part of this paper for commercial purposes without the written consent of the Brazilian Geophysical Society is prohibited.

\section{Abstract}

A análise espectral do tempo-frequência para as pulsações geomagnéticas Pc5 $(2-7) \mathrm{mHz}$, mostram uma considerável similaridade e simultaneidade entre as áreas geomagneticamente conjugadas. Os resultados sugerem que a faixa de $(2-4) \mathrm{mHz}$ são oscilações da ressonância da linha do campo geomagnético geradas por forças externas como a Instabilidade de KelvinHelmholtz. Os dados utilizados neste estudo foram obtidos em observatórios situados em áreas conjugadas e durante a tempestade magnética ocorrida em 25/outubro/2016. A análise foi realizada utilizando a transformada wavelet contínua e a coerência, nos registros da componente geomagnética horizontal.

\section{Introdução}

As pulsações geomagnéticas Pc5, com período entre (150 - $600 \mathrm{seg})$ são ondas ULF (Ultra Low Frequency) que estão constantemente presentes na magnetosfera perturbada. As Pc5 tem sido estudadas com dados de estações terrestres (PILIPENKO V. et al. 2010; OBANA Y. et al. 2005; HUDSON M.K. et al. 2004) e satélites (TAKAHASHI \& ANDERSON, 1992; BALASIS G. et al., 2015). Considera-se que as pulsações Pc5 estejam associadas com a Instabilidade de Kelvin-Helmholtz $(\mathrm{IKH})$ da fronteira magnetosférica, que são arrastadas pelo vento solar (BELAKHOVSKY e PILIPENKO, 2011; CAHILL L. e WINCKLER L., 1992). Estas Pc5 também podem estar relacionadas com o início súbito das tempestades SSC (storm sudden commencement) e impulsos súbitos SI (sudden impulses) (OLIVA D. et al., 2014; SAITO T. et al., 1976). Em geral, o período de pulsação pode ser encurtado ou alongado devido à compressão ou expansão da magnetosfera durante a SSC ou pelos SI negativos, mostrando comportamentos similares em pontos conjugados (NAGATA, T 1967; IWABUCHI M. et al., 1980; KATO K. 1994; KOZYREVA O. et al., 2006).

As pulsações Pc5 são controladas não apenas pela energia dos fenômenos gerados na magnetosfera, mas também pelas condições ionosféricas. (OBANA, Y. et al., 2005). A ionosfera pode exibir influências sob as pulsações geomagnéticas, ou seja, o sinal observado não corresponde à onda original, mas sim a uma onda eletromagnética irradiada por correntes induzidas na ionosfera, que sofrem uma significativa redução de amplitude e uma rotação de $90^{\circ}$ no eixo maior de polarização em relação ao sinal original (RESENDE A, 2018; KIVELSON, M. e RUSSELL, 1995).

Dois pontos na terra são "geomagneticamente conjugados" se estiverem em extremidades opostas de uma área geométrica de mesmo tubo de fluxo de campo geomagnético, estando cada extremidade nos hemisférios opostos, norte e sul (WESCOTT E. 1966). A componente horizontal $(\mathrm{H})$ do campo mostra-se mais susceptível às correlações e variações sob a influência de tempestades em postos conjugados (WESCOTT E., 1961)

Neste artigo apresentamos um estudo comparativo das pulsações magnéticas Pc5 detectadas simultaneamente em pontos conjugados, localizados em latitudes semelhantes sob influência de tempestade magnética com SSC. Foi utilizada a técnica da transformada wavelet para a análise espectral no domínio tempo-frequência.

\section{Metodologia}

Neste estudo, analisamos as pulsações magnéticas Pc5 de um par de observatórios magnéticos da rede INTERMAGNET (Real-time Magnetic Observatory Network) que estão geomagneticamente conjugados. As coordenadas geodésicas para esses observatórios estão apresentadas na tabela 1.

\begin{tabular}{|c|c|c|c|c|c|}
\hline \multirow[b]{2}{*}{ Observatórios } & \multirow[b]{2}{*}{ Pais } & \multicolumn{2}{|c|}{$\begin{array}{l}\text { Coordenadas } \\
\text { geográficas }\end{array}$} & \multicolumn{2}{|c|}{$\begin{array}{c}\text { Coord. de } \\
\text { pontos } \\
\text { conjugados }\end{array}$} \\
\hline & & Lat $\left[{ }^{\circ}\right]$ & Lon $\left[{ }^{\circ}\right]$ & Lat $\left[{ }^{\circ}\right]$ & Lon[] \\
\hline Kanoya (KNY) & Japão & 31,4 & 130,8 & $-15,8$ & 130,8 \\
\hline Kakadu (KDU) & Austrália & $-12,6$ & 132,4 & 28,2 & 132,5 \\
\hline
\end{tabular}

Tabela 1: Coordenadas geocêntricas dos observatórios magnéticos e seus pontos conjugados (para o ano 2016).

As coordenadas dos pontos conjugados foram calculadas empregando 0 modelo AACGM (Altitude adjusted corrected geomagnetic), que utiliza o IGRF-12 (Internal Geomagnetic Reference Field) para definir traços das linhas do campo magnético de um hemisfério a outro (Shepherd, S. G., 2014). O ponto conjugado do Kakadu (KDU) esta localizado a $3,5^{\circ}$ da direção sudeste do Kanoya (KNY), ver figura 1. Deve-se observar que os 
pontos conjugados estão sujeitos a deslocamentos sob efeito de tempestades magnéticas, entretanto a área de coerência conjugada (usando paramentos ionosféricos) é circular e de raio cerca de $700 \mathrm{~km}$ (WESCOTT et al.,1966). O raio de coerência que estamos considerando para este trabalho é de $5^{\circ}$ (figura 1 ).

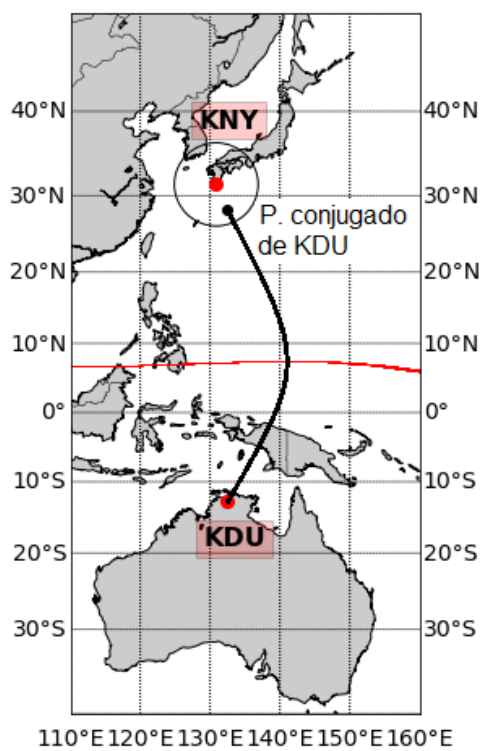

Figura 1: Mapa geográfico mostrando a localização dos observatórios e seus conjugados (pontos vermelhos e preto). A área de coerência (circulo preto) e o equador magnético (linha vermelha).

Os dados usados neste estudo correspondem a registros da componente $H$ do campo nos observatórios KNY e $\mathrm{KDU}$, com período de um minuto e registrados no dia 25 de outubro 2016. Este dia foi escolhido porque apresentou uma tempestade com índice magnético planetário considerável $(\mathrm{Kp}=7)$ e um inicio súbito da tempestade acontecido às 09:22 UT (figura 2).

Para selecionar as pulsações Pc5, os dados originais foram filtrados usando um filtro digital passa-faixa de resposta ao impulso infinita (IIR, Infinite Impulse Response). As propriedades das pulsações Pc5 em KNYKDU foram comparadas em termos da densidade espectral usando a análise da transformada wavelet contínua (CWT) e a coerência do sinal. A CWT de um sinal $x(t)$ é definida por (MUSAFAR L.M. 2014; DOMINGUES, 2016)

$$
W(b, a)=a^{-1 / 2} \int_{-\infty}^{+\infty} \psi^{*}\left(\frac{t-b}{a}\right) x(t) d t
$$

O parâmetro "a" se refere à escala e " $b$ " à translação da função wavelet analisadora $\psi^{*}$. A função de Morlet $(\gamma=3$,
TimeBandwidth $=60$ ) tem sido usada como a wavelet mãe, $\psi_{o}$ que se escreve:

$$
\psi_{0}(t)=\pi^{-1 / 4} \exp \left(i \omega_{0}\right) \exp \left(-t^{2} / 2\right)
$$

Onde $t$ descreve o tempo e $\omega_{0}$ é a frequência que representa o número de oscilações dentro a própria wavelet (DOMINGUES, 2016; MALLAT, 1999)

A transformada wavelet decompõe uma onda em suas componentes com base na frequência especificada por escalas wavelet. Isto permite monitorar quantitativamente a evolução dos sinais, decompondo uma série temporal em espaço de tempo-frequência. A potência da wavelet é determinada pelos coeficientes wavelet. Neste caso, a potência máxima das pulsações magnéticas Pc5 em um segmento horário pode ser representada pelo máximo do espectro total de energia para todas as escalas wavelet (MUSAFAR L.M. 2014; OLIVA D. et al., 2014).

Para complementar nosso estudo calculamos a coerência quadrada de wavelet das Pc5. A coerência wavelet é uma estatística que pode ser usada para examinar e quantificar a relação entre dois sinais ou conjuntos de dados (MALLAT, 1999). A coerência wavelet de duas séries temporais $x$ e $y$ é:

$$
R_{n}^{2}(s)=\frac{\left|S\left(s^{-1} W_{n}^{x y}(s)^{2}\right)\right|^{2}}{\left.\left.S\left(s^{-1}\left|W_{n}^{x}(s)\right|^{2}\right) \cdot S\left(s^{-1} \mid W_{n}^{y}(s)\right)\right|^{2}\right)}
$$

Onde $S$ é um operador de suavização no tempo e na escala. Observe que essa definição se assemelha ao de um coeficiente de correlação tradicional. A coerência wavelet fornece um estimador qualitativo da evolução temporal do grau de linearidade da interação entre duas séries temporais numa dada escala. $O$ valor um significa uma relação linear entre os sinais (DOMINGUES et al., 2016). Escreva o operador de suavização $S$ como:

$$
S(W)=S_{\text {escala }}\left(S\left(_{\text {tempo }} W_{n}(s)\right)\right)
$$

Onde $S_{\text {escala }}$ denota o alisamento ao longo do eixo da escala wavelet e $S_{\text {tempo a suavização no tempo }}$ (GRINSTED et al., 2004; MALLAT, 1999).

\section{Resultados}

A figura 2 mostra a variação diurna da componente horizontal, dos observatórios KNY e KDU. 


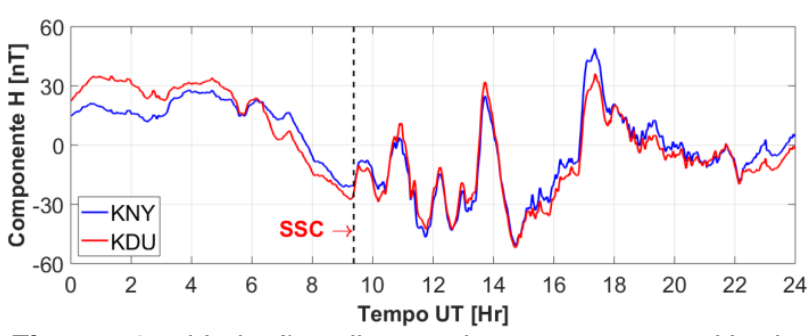

Figura 2: Variação diurna da componente $H$ dos observatórios conjugados KNY -KDU (25/Out/2016).

As curvas de variações de $\mathrm{H}$ nos observatórios mostram um comportamento similar. Este comportamento é mais semelhante depois da presença da SSC (09:22 UT). Esta semelhança, em forma e amplitude, sugere que as duas áreas estão interligadas pelas linhas do campo magnético, confirmando a área de coerência conjugada escolhida. Semelhança das curvas do campo magnético em áreas conjugadas tem sido encontrada em trabalhos anteriores (WESCOTT, E. 1961, NAGATA, T. 1967)

As pulsações Pc5 obtidas nos pontos conjugados depois de filtrar as componentes $\mathrm{H}$ são apresentadas na figura 3. Para ter mais detalhes da resposta do campo durante a tempestade magnética e a SSC, foi seccionado um intervalo entre 10:00 e 20:00 UT.

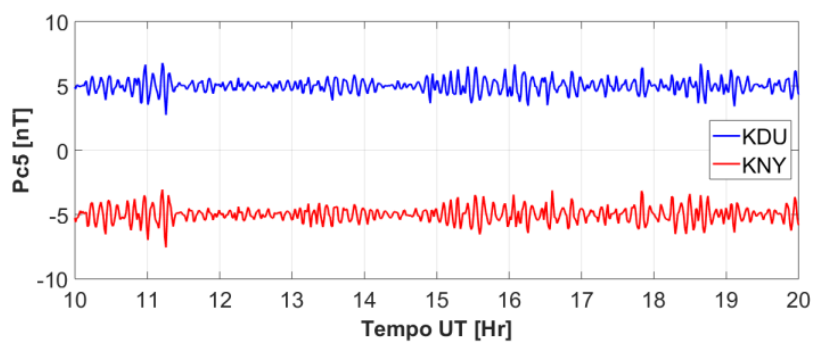

Figura 3: Dados filtrados mostrando pulsações magnéticas Pc5 registradas nos dados dos observatórios conjugados KNY-KDU (25/Out/2016).

As curvas das pulsações Pc5 no KNY e KDU mostram um padrão de propagação consistente, isto é, pacotes de ondas Pc5 simultâneas no tempo e com amplitudes similares (3 nT), especialmente, ao redor das 11:00 UT e entre às 15:00-16:30 UT. Estudos prévios também mostraram comportamento similar das Pc5 em pontos conjugados (OBANA et al., 2005; TONEGAWA et al., 1984)

A figura 4 mostra os espectros de potência de wavelet para os dois observatórios e a coerência wavelet entre eles. Nos espectrogramas de KNY e KDU (figura 4) podese verificar que a principal contribuição para a potência espectral está localizada na faixa de frequências de $2,2 \mathrm{mHz}$ a $4 \mathrm{mHz}$, ou de 250 a $450 \mathrm{~s}$, que pertence à zona de frequência contendo o limite inferior das pulsações
Pc5. Também foi verificado que os máximos valores da transformada, ao redor das 11:00 UT, assumem valores muito parecidos em ambos gráficos da wavelet. Os resultados destas indicam que o evento da tempestade foi simétrico para os observatórios, especialmente ao redor das 11:00 UT.

No gráfico da coerência das wavelet, a cor amarela indica a máxima coerência verificada entre os espectrogramas da wavelet (KNY-KDU), que corresponde a 1. Este gráfico mostra uma grande coerência entre as Pc5 nos pontos conjugados, a maioria da área do gráfico contém o máximo valor da coerência.

Foi verificado que a máxima coerência entre os observatórios acontece ao redor de 10:00 e 11:30 UT e entre as frequências de 2 a $4 \mathrm{mHz}$. O gráfico da coerência quadrado de wavelet mostra outro evento considerável ocorrido ao redor das 15:30 e 16:30 UT. A alta coerência pode indicar a geração das Pc5 em presença da tempestade, e como ela pode influenciar simultaneamente nos pontos conjugados através das linhas de força associada.

As pulsações Pc5 em KDY e KDU mostraram caraterísticas espectrais similares em: amplitude, período, intensidade, forma das ondas e estrutura das wavelet (Figura 2, 3, 4). Isso pode indicar, especificamente que depois do inicio súbito da tempestade, entre as 10:00-11:30 UT, que a fonte das pulsações estava localizada no plano equatorial da magnetopausa, e as ondas propaga-se através as linhas de fluxos até os pontos conjugados. Em contra partida, as pequenas diferenças mostradas nos gráficos wavelet, ex: entre as 15:30 e 16:30 UT, podem estar associadas à diferenças ionosféricas locais e possíveis reflexões das ondas Pc5 na parte superior da ionosfera

\section{Conclusões}

Pelos resultados foi verificado que as áreas conjugadas dos observatórios KNY e KDU, as curvas das variações da componente horizontal e as pulsações geomagnéticas Pc5 são semelhantes em amplitude, estrutura de onda e duração. Além disso, os escalogramas de wavelets mostraram que as potências espectrais tem padrões similares e simultâneos no tempo, e sua coerência wavelet é máxima ao redor das 11:00 UT. Dos resultados obtidos, pode-se concluir que existe uma boa correlação entre a atividade magnética nas áreas conjugadas. Esta correspondência indica que as Pc5 se propagaram através das linhas de indução do campo até suas extremidades opostas, sendo observadas simultaneamente nos pontos conjugados. 

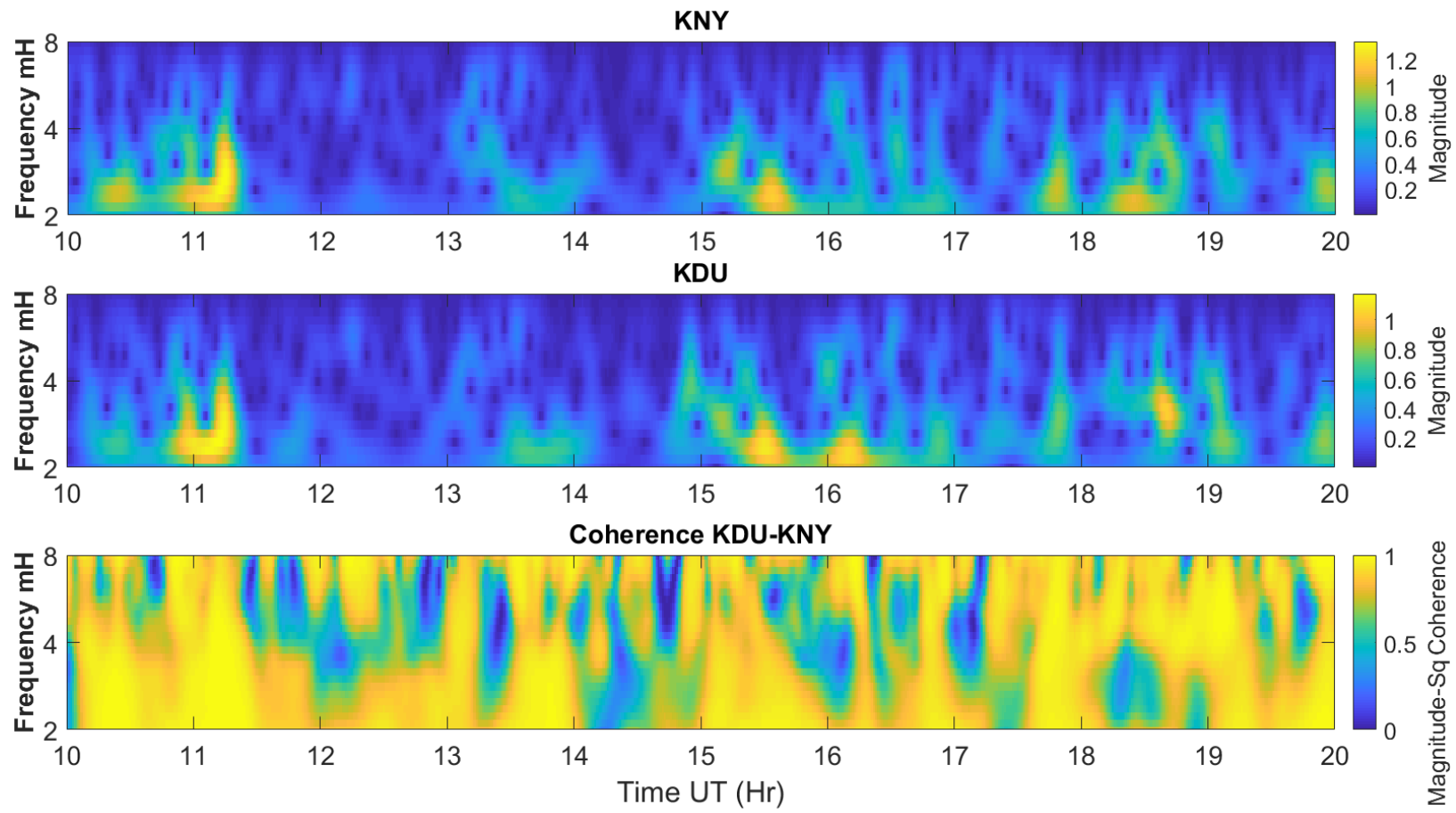

Figura 4: Espectrogramas de wavelet no KNY e KDU, e a coerência entre eles.

Nós sugerimos que a instabilidade de Kelvin-Helmholtz esta relacionada com o mecanismo de geração das Pc5. Outro fato importante de descrever é algumas pequenas diferenças nos escalogramas de wavelets, que são resultados da interação das Pc5 com a ionosfera de cada área conjugada. A ionosfera de cada ponto conjugado têm características desiguais, que pode variar para latitudes diferentes.

Estamos conscientes de que é difícil realizar um estudo sobre o comportamento das pulsações em pontos conjugados usando apenas um par de observatórios. Apesar deste fato, mostramos que algumas informações podem ser extraídas processando os dados desses observatórios magnéticos. A inclusão de mais observatórios será considerada para futuros estudos

\section{Agradecimentos}

Agradecemos a INTERMAGNET pelos dados dos observatórios usados neste estúdio. Também a CAPES pela bolsa de doutorado.

\section{Referencias}

BALASIS, G., et al. "Multi-satellite study of the excitation of Pc3 and Pc4-5 ULF waves and their penetration across the plasmapause during the 2003 Halloween superstorm." Annales Geophysicae. Vol. 33. No. 10. Copernicus $\mathrm{GmbH}, 2015$.

BELAKHOVSKY, V., PILIPENKO, V. "Generation of magnetic eld Pc5 pulsations and particle fluxes during the recovery phase of a magnetic storm on October 31 , 2003", Geomagnetism and Aeronomy, v. 51, n. 5, pp. 599. 2011.

CAHILL, L., WINCKLER, J., "Periodic magnetopause oscillations observed with the GOES satellites on March 24, 1991", Journal of Geophysical Research: Space Physics, v. 97, n. A6, pp. 8239-8243. 1992.

DOMINGUES, M. O., et al. "Explorando a transformada wavelet contínua." Revista Brasileira de Ensino de Física 38.3. 2016.

FUKUSHIMA, N., 1969, "Equivalence in Ground Magnetic Effect of Chapman-Vestines and Birkeland-Alfven's Electric Current-Systems for Polar Magnetic Storms", Rept. Jonos. Space Res. Japan, v. 23, pp. 219-227, 1969. 
GRINSTED, ASLAK, JOHN C. MOORE, and SVETLANA JEVREJEVA. "Application of the cross wavelet transform and wavelet coherence to geophysical time series." Nonlinear processes in geophysics 11.5/6. 2004.

HUDSON, M. K., et al. "A study of Pc-5 ULF oscillations." Annales Geophysicae. Vol. 22. No. 1. 2004.

IWABUCHI, M., FUKUNISHI H., and SATO N. "Spectral and Polarization Characteristics of Pc 3-5 Magnetic Pulsations Observed at the Syowa Station-Iceland Conjugate Pair (ULF Waves in the Magnetosphere)(Proceedings of the Second Symposium on Coordinated Observations of the lonosphere and Magnetosphere in the Polar Regions: Part I)". 1980.

KATO, KEN-ICHI, NATSUO SATO, and YUTAKA TONEGAWA. "Estimation of Geomagnetic Conjugate Points Using Pc5 Geomagnetic Pulsation." Journal of geomagnetism and geoelectricity 46.5. 1994

KIVELSON, M. G.; RUSSELL, C. T. "Introduction to space physics". Cambridge: Cambridge University Press, 1995.

KOZYREVA, O. V., et al. "Long-period geomagnetic pulsations in the quasi-conjugate arctic and antarctic regions during the magnetic storm of April 16-17, 1999." Geomagnetism and Aeronomy 46.5. 2006.

MALLAT, STÉPHANE. "A wavelet tour of signal processing". Elsevier, 1999.

MUSAFAR, L. MUHAMMAD K., et al. "Pc5 magnetic pulsations during the outer electron radiation belt." International Journal of Technology 5.3, 269-276, 2014.

MCPHERRON, R. L., "Magnetic pulsations: their sources and relation to solar wind and geomagnetic activity", Surveys in Geophysics, v. 26, n. 5, pp. 545-592, 2005.

NAGATA, T. "Geomagnetic Conjugacy between the Antarctic and the Arctic." 65-80, 1967.

OBANA, Y. et al. "North-south asymmetry of the amplitude of high-latitude $\mathrm{Pc} 3-5$ pulsations: Observations at conjugate stations." Journal of Geophysical Research: Space Physics 110.A10, 2005.

RESENDE A. "análise de pulsações magnéticas pc3 e pc4 na região da anomalia magnética do atlântico sul". Teses de mestrado, INPE, 2018.
OLIVA, DAVID, MARCELA C. MEIRELLES, and ANDRÉS RR PAPA. "A Study of Pc4-5 Geomagnetic Pulsations in the Brazilian Sector." arXiv preprint arXiv:1404.4321, 2014.

PILIPENKO, V., et al. "Generation of magnetic and particle Pc5 pulsations during the recovery phase of strong magnetic storms." Proceedings of the Royal Society A: Mathematical, Physical and Engineering Sciences 466.2123, 2010.

SAITO, T. and MATSUSHITA, S. "Geomagnetic Pulsations Associated with SSC and SI" Planetary Space Sci. 15, p.573. 1967.

TAKAHASHI, K., and R. L. McPherron (1982), Harmonic structure of Pc 3- 4 pulsations, J. Geophys. Res., 87, 1504.

TONEGAWA, Y., and $\mathrm{H}$. FUKUNISHI. "Harmonic structure of Pc 3-5 magnetic pulsations observed at the Syowa-Husafell conjugate pair." Journal of Geophysical Research: Space Physics 89.A8, 1984.

WESCOTT, E. "Magnetoconjugate phenomena." Space Science Reviews 5.4, 1966.

WESCOTT, E. "Magnetic variations at conjugate points." Journal of Geophysical Research 66.6, 1961. 